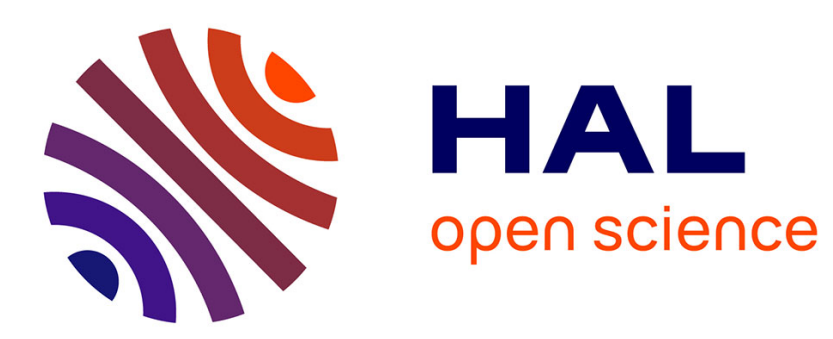

\title{
The influence of the viscosity ratio on polymer droplet collision in quiescent blends \\ Claude Verdier
}

\section{To cite this version:}

Claude Verdier. The influence of the viscosity ratio on polymer droplet collision in quiescent blends. Polymer, 2001, 42, pp.6999-7007. hal-00322140

\section{HAL Id: hal-00322140 https://hal.science/hal-00322140}

Submitted on 16 Sep 2008

HAL is a multi-disciplinary open access archive for the deposit and dissemination of scientific research documents, whether they are published or not. The documents may come from teaching and research institutions in France or abroad, or from public or private research centers.
L'archive ouverte pluridisciplinaire HAL, est destinée au dépôt et à la diffusion de documents scientifiques de niveau recherche, publiés ou non, émanant des établissements d'enseignement et de recherche français ou étrangers, des laboratoires publics ou privés. 


\title{
The influence of the viscosity ratio on polymer droplet collision in quiescent blends
}

\author{
C. VERDIER
}

Laboratoire de Rhéologie*

1301 rue de la piscine

BP53 - Domaine Universitaire

38041 Grenoble Cedex 9

France

Fax. (33) $476825164 \quad$ e-mail : verdier@ujf-grenoble.fr

\begin{abstract}
The coalescence of polymer droplets is studied, in particular the final regime named collision. This phenomenon involves the complex deformation of the interface, a motion driven by the interfacial tension, and is mainly governed by the viscosity ratio. An accurate measurement technique of the collision time has been explored experimentally. In a recent paper [C. Verdier, C. R. Acad. Sci. Paris Série IV, 2000], a particular case of the collision was studied, corresponding to viscosities in the same range. One of the aims of the present work is to analyze collision, and to extend these previous results to a wider range of viscosity ratios. For droplets of equal size, three regimes are obtained, corresponding to power law dependence of the collision time as a function of the viscosity ratio.

The flow field shows the importance of the area close to the neck, where most of the flow dissipations occur. By assuming that this flow gives rise to a competition between interfacial energies and shear viscous dissipations, a simple model is presented, based on a previous study [Brochard-Wyart F, de Gennes PG, Advances in Colloid and Interface Science, 1992], and it recovers two of the experimental regimes. The final one can also be predicted using results from Frenkel [J. Phys. USSR, 1945]. This theory is in qualitative agreement with the observed data.

This experimental technique is simple and provides a new way to measure interfacial tension, when the viscosities of the two fluids are known. The advantage of the technique is that sample preparation is easy, and that it requires only an optical microscope.
\end{abstract}

(Keywords : coalescence, collision, droplet, viscosity ratio, interfacial tension)

\footnotetext{
${ }^{*}$ Université de Grenoble I, Institut National Polytechnique de Grenoble, CNRS (UMR 5520)
} 


\section{INTRODUCTION}

The mixing of two polymers is not quite fully understood, because it requires wellcontrolled mixers, and the geometry of the blades is generally not simple. Complex flows result from such mixing and it becomes hard to control the size and dispersion of the droplets of one polymer into the other. As mixing goes on, coalescence and break-up of the droplets can happen simultaneously, although they have reasons for appearing respectively at small or high shear velocities. In fact such motions are combinations of both shear and elongational flows, depending on the point in the fluid mixture. Therefore, the application of simple flows is a first step towards the understanding of the mixing process.

Taylor [1] studied well-defined flows such as shear and elongational ones, computed the droplet deformation, and found criteria for break-up. Grace [2] also paid attention to ideal flow situations and studied the critical conditions for break-up and coalescence over a very wide range of viscosity ratios. The break-up problem was again considered in shear and elongational flows using an isolated drop [3-4]. Recently, a few authors [5-6] used microscopy to control droplet size when shearing a polymer emulsion. This situation shows that critical regimes for coalescence [7] and break-up [1] can be established. The latter results are obtained by using a distribution of droplets in a moving fluid, and one assumes a certain probability of encounter for the coalescence process. For example, coalescence requires that a sufficient amount of time ( $t_{e}$, interaction time) is necessary for the encounter to take place, therefore an example of such a probability is $\mathrm{P}=\exp \left(-\mathrm{t}_{\mathrm{d}} / \mathrm{t}_{\mathrm{e}}\right)$, if $\mathrm{t}_{\mathrm{d}}$ is the time necessary to drain the interstitial fluid. If the interaction time is long enough, then coalescence occurs $(\mathrm{P} \approx 1)$, otherwise the drainage is not completed $(\mathrm{P} \approx 0)$. These ideas are reviewed for example in Chesters' paper [8]. 
Let us now consider the ideal situation of a coalescence process with only two droplets. Three parts can be distinguished : the first one is the drainage of the interstitial fluid $[8,9]$, which is very important and controls the total coalescence time. The second one is related to the opening of a tiny hole which leads to collision. Typically, this process occurs when the gap between the two droplets is smaller than a certain distance (usually about $50 \mathrm{~A}$ ), which is probably fluid dependent. The last stage is the collision (or fusion) which corresponds to the merging of the two droplets, which is of interest here, and has not been studied so much in the literature.

This situation has only been considered before in special cases, like for the flow of crystalline solids under the action of surface tension [10] and in the sintering of metallic powders [11]. A theoretical approach [12-13] based on mapping functions has also been found but can be applied for the two-dimensional case only. Finally, surface diffusion has also been studied through computations of the initial rapid process [14], showing the initial time dependence. Another recent approach [15] has analyzed the collision process when varying the viscosity ratio of the two fluids, thus showing a dependence of the collision time on the geometric mean $\sqrt{\eta_{1} \eta_{2}}$ of the two viscosities ( $\eta_{1}$ for the droplet and $\eta_{2}$ for the matrix). This latter work considers the collision as well as the evolution of the neck region, i.e. the point of largest curvature. The viscosity ratio has been varied over two decades, which corresponds to situations where the fluid viscosities are not so different. The fluids used were polymers at small enough rates so that the elasticity effects are negligible. Other studies can be found in the literature concerning the effect of viscoelasticity [16] and the role of copolymers [17] at the interface during coalescence, but are not the purpose here.

In this work, the case of two droplets is considered, bearing in mind the main application, which is the understanding of the dynamics of a polymer emulsion. An overall picture of the role of the viscosity ratio on droplet collision is carried out, with the aim of 
determining new regimes compared to the previous study [15]. In the first part, the experiment is recalled and new results are presented, over a very large viscosity ratio range (six decades). Three typical regimes are obtained. In the next part, the corresponding theory is proposed, based on the work of Brochard and de Gennes [18]. Also we made use of a previous result by Frenkel [10] to explain some of the data. A discussion is proposed next, and the application of this technique as a new interfacial tensiometer is suggested. This technique may be very adequate for the investigation of polymer systems.

\section{EXPERIMENTAL PART}

The experimental system that was used for the observation of collision of the droplets is rather simple and has been presented before [15]. Therefore we will briefly summarize the method here, as well as the automated technique coupled with it. This technique has been used in order to reduce time consuming data processing. Also, the fluids that were used will be presented. Let us start with the experimental set-up.

\section{Set-up}

An emulsion is first made after mixing two polymers for about two minutes. The mixing can be done manually for example, since the polymer are not too viscous. The system is then set to rest so that the air entrapped can be released. A small sample is taken from the mixture and put between a plate and a micro cover-glass. Observations are simply made with an optical microscope (Leica, DML model) using a magnification of x20 or x 50 , depending on droplet size (usually the radius varies between $5 \mu \mathrm{m}$ and $30 \mu \mathrm{m}$ ). One needs to locate two droplets of approximately equal size, therefore there is a need for a sufficient number of droplets in the blend. This can be easily achieved by mixing enough amounts of polymer 1 
inside polymer 2. Once the droplets are located and are close enough, the coalescence process can be visualized. The interstitial fluid 2 is slowly removed (drainage) until the drops actually come very close to each other. Sometimes, a flattening effect is observed, as described previously [8]. Finally a tiny hole is opened, and the collision process takes place. A video camera (3-CCD color, KY-F55B JVC model, 25 frames/s) is coupled to the microscope and films are made on a U-matic videorecorder (SP type, VO-9600P model). Photographs can be downloaded and measurements are made on a PC computer using the dedicated Labview ${ }^{\mathrm{TM}}$ software. A typical experiment is depicted in figures 1a-1f. Figure 1a shows two droplets of roughly equal radii getting very close together. In figure $1 b$, the droplets are touching and a hole is opening. After some time (figure 1c), the droplets have started to coalesced, until they look like one larger deformed droplet (figure 1d). The final stages are next shown while the fluids relax and longer times are needed (figures 1e) until the composite droplet finally reaches the shape of a sphere (figure 1f).

\section{System parameters}

The system we are looking at (figure 2) is an axisymmetric two-droplet system. Two droplets (fluid 1) are imbedded into a matrix (fluid 2). The densities of inner and outer fluid are respectively $\rho_{1}$ and $\rho_{2}$. Their viscosities are respectively $\eta_{1}$ and $\eta_{2}$. The other parameters of the problem are droplet radii $R_{1}$ and $R_{2}$ and interfacial tension $\sigma . L(t)$ is the distance between the droplets, is time dependant and decreases as time goes on. In this case, van der Waals forces act to bring the droplets close to each other.

To simplify, we will be looking at almost equal sized droplets, so that $\mathrm{R}_{1}=\mathrm{R}_{2}$. Two such droplets can be found because the system is a dispersion with a large number of droplets. In this problem, a relevant dimensional analysis [15] shows that the only important parameters are the characteristic time of collision $t_{c}$ (defined more precisely below), in its 
dimensionless form $\sigma t_{\mathcal{c}} / \eta_{1} R_{1}$, and the viscosity ratio $\eta_{1} / \eta_{2}$. Indeed the Suratman number $\mathrm{S}=\sigma \rho_{1} \mathrm{R}_{1} / \eta_{1}^{2}$ can be neglected because of the high viscosities $(>1 \mathrm{~Pa} . \mathrm{s})$ of the fluids and gravity effects are also negligible in the experiment. Wall effects can also be neglected, by choosing droplets in the center of the fluid (between the plate and micro-cover glass). Let us also note that a typical velocity $V$ is $R_{1} / t_{c}$ so that $\sigma t_{c} / \eta_{1} R_{1}$ can also be interpreted as the inverse of the capillary number. Therefore we are looking at the dependence of the group $\sigma t_{c} / \eta_{1} R_{1}$ as a function of the viscosity ratio $\eta_{1} / \eta_{2}$. Fluid polymers with various viscosities will be chosen so that a large number of decades can be covered in terms of $\eta_{1} / \eta_{2}$.

\section{Polymers}

The fluids are a set of silicones or polydimethylsiloxanes (PDMS, Rhône-Poulenc, 47VT series, viscosities at 25C: 1 Pa.s, 10 Pa.s, 30 Pa.s, 60 Pa.s, 100 Pa.s, 200 Pa.s, 1000 Pa.s) and polyisobutylenes (PIB, D-series, BP-Chimie, viscosities at $25^{\circ} \mathrm{C}: 38$ Pa.s, 130 Pa.s, 430 Pa.s). The main advantage of these fluids is that they are melts at room temperature, and they have close enough refraction indexes (1.5 for PDMS and 1.41 for PIB) so that one can observe PDMS in PIB and vice versa. Note that this system has been used by several authors previously [5-6]. It will then be possible to cover a large range of viscosity ratio, basically ranging from $10^{-3}$ to $10^{3}$, in other words over 6 decades, which is equivalent to data obtained by Grace [2] for experiments on break-up. These polymers are known to have quite short relaxation times (a few milliseconds) therefore they behave like newtonian liquids.

\section{Collision time $\left(t_{c}\right)$}

The characteristic time of collision will be defined as follows. As seen from observations in figure 1a-1f, the initial starting time of the collision is hard to determine, therefore, we avoid to define a time zero. It is better to plot the total length of the composite 
droplet $\mathrm{D}(\mathrm{t})$ (figure 3 ) and determine the curve $\mathrm{D}(\mathrm{t}) / \mathrm{D}_{0}$ versus time $\mathrm{t}$, where $\mathrm{D}_{0}=2\left(\mathrm{R}_{1}+\mathrm{R}_{2}\right)$. This curve shows an inflexion point at time $t_{\mathrm{i}}$. The inverse of the negative of the slope, which is homogeneous to a time will be defined as the collision time $t_{c}=-D_{0} / D^{\prime}\left(t_{i}\right)$, where $D^{\prime}(t)$ is the time derivative of $\mathrm{D}(\mathrm{t})$. This is shown in figure 3 , in the most general case where $\mathrm{R}_{1} \neq \mathrm{R}_{2}$. One needs to remember that this relation just gives a very precise (mathematical) way to find $t_{c}$. Indeed, $D(t) / D_{0}$ actually ranges between 1 and $R_{e} /\left(R_{1}+R_{2}\right)$, where $R_{e}{ }^{3}=R_{1}{ }^{3}+R_{2}{ }^{3}$ from mass conservation. In our case where $R_{1}=R_{2}, R_{e}=2^{1 / 3} R_{1}$. Then $D(t) / D_{0}$ decreases from 1 to $2^{-2 / 3} \approx 0.63$. This means that a more realistic coalescence time would be more likely in the range of $0.37 \mathrm{t}_{\mathrm{c}}$.

Anyway, this definition is convenient because it is easy to determine $t_{c}$ experimentally and this definition will be used throughout this paper.

\section{Automated determination of $t_{c}$}

Since a lot of experiments are required, in order to cover six decades, we choose an automated system for the measurements and the determination of $t_{c}$. Videos are stored on a PC computer, and the required frames are kept to be analyzed later. Enough data points are chosen so that the rapid decay of $\mathrm{D}(\mathrm{t})$ can be monitored accurately. The distance $\mathrm{D}(\mathrm{t})$ is measured by pointing a line going through the droplets axis and the Labview ${ }^{\mathrm{TM}}$ software enables to get the distance easily, after a calibration procedure has been used. One can then plot the ratio $D(t) / D_{0}$ versus time. We choose to fit the data with the following function $\mathrm{F}(\mathrm{t})=\mathrm{D}(\mathrm{t}) / \mathrm{D}_{0}$

$$
\mathrm{F}(\mathrm{t})=\frac{\mathrm{m}_{1}+\mathrm{m}_{2} \mathrm{t}^{2}}{\mathrm{~m}_{1}+\mathrm{t}^{2}}
$$

This function is a good candidate (figure 4 ) because it starts from $1(\mathrm{t}=0)$, and tends to $\mathrm{m}_{2}$ $(t \rightarrow \infty)$. The two parameters $m_{1}\left(s^{2}\right)$ and $m_{2}$ (dimensionless) can be adjusted using simple 
fitting tools available on classical graphics software. It is to be checked that $\mathrm{m}_{2}$ is of the order 0.63 (see previous subsection). Also it is found that the curve $F(t)$ is always in good agreement with the experimental data (see figure 4).

The other reason for choosing this function is that it shows of course an inflexion point, just like in the experiments. After doing some simple algebra, we find that the inflexion time $t_{i}$ is:

$$
\mathrm{t}_{\mathrm{i}}=\sqrt{\frac{\mathrm{m}_{1}}{3}}
$$

and the slope at the inflection point $F^{\prime}\left(t_{i}\right)=-1 / t_{c}$ provides the required value of the collision time $t_{c}$, once the determination of the two parameters $m_{1}$ and $m_{2}$ has been made using the automated technique :

$$
\mathrm{t}_{\mathrm{c}}=\frac{8 \sqrt{\mathrm{m}_{1} / 3}}{3\left(1-\mathrm{m}_{2}\right)}
$$

Figure 4 shows a set of data, as well as the errors involved in the fitting procedure. The values of the two parameters are found numerically, by optimal fitting. We check that $m_{1}$ is positive and that $\mathrm{m}_{2}$ is close to 0.63 , which validates our technique. This systematic determination of the collision time will be used in all the experiments.

\section{Experimental results}

The main results of the experimental part of this work are shown in figure 5. The plot of the dimensionless collision time $\sigma t_{c} / \eta_{1} R_{1}$ as a function of the viscosity ratio $\eta_{1} / \eta_{2}$ is given and reveals three different regimes, which correspond to well-defined slopes of $-1,-0.5$ (roughly) and 0 . From the data we can exhibit three formulae for power-law relationships.

$$
0.001<\eta_{1} / \eta_{2}<0.1 \text { slope }-1 \quad \sigma t_{c} / \eta_{1} R_{1} \cong 12\left(\eta_{1} / \eta_{2}\right)^{-1} \text { or } t_{c} \cong 12 \eta_{2} R_{1} / \sigma
$$




$$
\begin{aligned}
& 0.1<\eta_{1} / \eta_{2}<10 \quad \text { slope }-1 / 2 \quad \sigma t_{c} / \eta_{1} R_{1} \cong 40\left(\eta_{1} / \eta_{2}\right)^{-0.5} \text { or } t_{c} \cong 40\left(\eta_{1} \eta_{2}\right)^{0.5} R_{1} / \sigma \\
& 10<\eta_{1} / \eta_{2}<1000 \quad \underline{\text { slope } 0} \quad \sigma t_{c} / \eta_{1} R_{1} \cong 12\left(\eta_{1} / \eta_{2}\right)^{0} \quad \text { or } t_{c} \cong 12 \eta_{1} R_{1} / \sigma
\end{aligned}
$$

In these formulae, $\sigma$ is in $N / m, t_{c}$ is in $s, \eta_{1}$ is in Pa.s and $R_{1}$ is in $m$.

We recover the previous result [15] with the slope of -0.5 which corresponds to a collision time varying like $\sqrt{\eta 1 \eta 2}$ in other words like the geometric mean of the viscosities. Two new regimes are obtained which correspond to collision times varying like the highest viscosity. These regimes seem to be symmetric, because the corresponding constants, equal to approximately 12 (no dimension in formulae $4 \mathrm{a}$ and $4 \mathrm{c}$ ) are the same. This result will be discussed in the next sections.

\section{THEORY}

\section{General framework}

Let us look at the geometry of the problem. Two spheres of equal radii collide together and form a new droplet, whose shape can be approximated within good accuracy to the reunion of two spheres (see figure 6). We define the angle $\theta$ which is convenient for the calculations. $\theta$ is a function of time t. From mass conservation, and using the sphere approximation, we can recover the following result due to Frenkel [10], by considering the volume of each part of the spheres: indeed, the fluid density $\left(\rho_{1}\right)$ is assumed to be a constant therefore the volume of the new deformed drop is a constant equal to $8 / 3 \pi R_{1}{ }^{3}$ (the initial volume of both droplets). If $\mathrm{R}(\theta)$ is the radius of each of the new spheres (at the beginning of collision), then we find, after some geometry:

$$
R(\theta)=R_{1}\left(\frac{4}{2+3 \cos \theta-\cos ^{3} \theta}\right)^{1 / 3}
$$


Note that $\mathrm{R}(\theta)$ is measured from the center of the spheres (figure 6), and that this center a moving. Only the center of mass of the spheres is fixed.

The velocity $\mathrm{V}$ at the cusp (see figure 6) can also be calculated as a function of geometry

$$
\mathrm{V}=\overline{\mathrm{R} \sin \theta}
$$

Formula (6) together with the use of (5) can be combined to obtain the rates $\dot{R}$ and $\dot{\theta}$, after some algebra:

$$
\begin{aligned}
& \dot{\mathrm{R}}=\mathrm{V} \frac{\sin ^{3} \theta}{(1+\cos \theta)^{2}} \\
& \dot{\theta}=\frac{\mathrm{V}}{\mathrm{R}} \frac{2+3 \cos \theta-\cos ^{3} \theta}{(1+\cos \theta)^{2}}
\end{aligned}
$$

Note that $\mathrm{V}$ is actually a function of $\theta: \mathrm{V}=\mathrm{V}(\theta)$. It is likely that $\mathrm{V}$ starts with a maximum as $\theta=0$ and decreases as $\theta$ increases. Indeed, the driving force (due to interfacial tension) is stronger when the radius of curvature is smaller. We are mainly concerned with the case of small $\theta$, since we are trying to describe the first instants of the collision process, in order to find the characteristic collision time $t_{c}$ (steep part of the curve $D(t) / D_{0}$, see figure 3 ). Other important parameters are the distance $\mathrm{x}(\theta)$ and length $\mathrm{D}(\theta)$ :

$$
\begin{aligned}
& x(\theta)=R(\theta) \sin \theta \\
& D(\theta)=2 R(\theta)(1+\cos \theta)
\end{aligned}
$$

Since we are looking at the first stages (rapid) of the collision process, we can make use of the following assumptions, to first order in $\theta$ :

$$
\mathrm{R}(\mathrm{t}) \approx \mathrm{R}_{1} \quad \mathrm{x} \approx \mathrm{R}_{1} \theta \quad \mathrm{D} \approx 4 \mathrm{R}_{1} \quad \dot{\mathrm{R}} \approx \mathrm{V} \theta^{3} / 4 \quad \dot{\theta} \approx \mathrm{V} / \mathrm{R}_{1}
$$

This means that the collision time $t_{c}$ will be vary as $\theta_{0} R_{1} / V$, where $\theta_{0}$ is some angle to be determined. In addition, the rate of change of $D(t)$, designed by $\dot{\mathrm{D}}$, can be approximated by 


$$
\dot{\mathrm{D}} \approx-2 \mathrm{~V}(\theta) \theta
$$

From figure 3 , which provides the collision time definition, we notice that $t_{c}$ actually corresponds to the maximum slope of $\mathrm{D}(\mathrm{t})$ or $\mathrm{D}(\theta)$, in other words to the value $\theta_{0}$ of the angle for which the maximum of the product $\mathrm{V}(\theta) \theta$ is obtained. This will be used to predict the typical velocity $\mathrm{V}\left(\theta_{0}\right)$.

\section{Energy balance}

The flow involved inside and outside the droplet is rather complex, therefore, we will assume that most of the energy is produced by the driving force, i.e. interfacial tension, and dissipated through viscous flows in both fluids 1 and 2 . We will make use of the ideas of de Gennes and Brochard [18-19], who studied the velocity profile inside the corner defined by a plane and an interface with a small angle [18]. Figure 7 is a good representation of the velocity field. We assume that the angle $\theta$ is small. The profile is assumed to be parabolic, due to the lubrication hypothesis in that region of flow. This is valid in this case because, at the beginning of motion, there exists a very small gap between the droplets.

To determine the maximum slope from (12), we need to find the relationship $V(\theta)$. Point A has velocity $\mathrm{V}(\theta)$. At point $\mathrm{B}$, there are two components: $\mathrm{V}_{\mathrm{N}}$ and $\mathrm{V}_{\mathrm{T}}$ are respectively the normal and tangential components (with respect to the interface) of the velocity at B. There are different types of shear which need to be considered, which will lead to dissipation. We approximate $\mathrm{V}_{\mathrm{N}}$ by

$$
\mathrm{V}_{\mathrm{N}} \approx \mathrm{V} \theta
$$

where $\theta$ is considered to be rather small (less than $30^{\circ}$ means the error is less that $5 \%$ ).

$\mathrm{V}_{\mathrm{T}}$ can be obtained by writing the continuity of the shear stress at point $\mathrm{B}$, i.e. across the interface: $\eta_{1} V_{T} / h=\eta_{2} V / h \theta$, therefore 


$$
\mathrm{V}_{\mathrm{T}} \approx \frac{\eta_{2}}{\eta_{1}} \frac{\mathrm{V}}{\theta}
$$

Within polymer 1 , there are two kinds of shear: $\frac{\partial V_{\mathrm{Z}}}{\partial \mathrm{x}} \approx \frac{\mathrm{V} \theta}{\mathrm{h}}$ and $\frac{\partial \mathrm{V}_{\mathrm{x}}}{\partial \mathrm{z}} \approx \frac{\mathrm{V}_{\mathrm{T}}}{\mathrm{h}}$.

Within polymer 2 , the shear $\frac{\partial V_{x}}{\partial z}$, from Poiseuille flow, is of the order $\frac{V}{\theta h}$.

From [18], we write the total dissipation $\mathrm{D}_{\mathrm{v}}$ in the system, as the product of the viscosity and the square of the shear rate times the area of concern:

$$
D_{\mathrm{v}}=\eta_{1}\left\{(\mathrm{~V} \theta / \mathrm{h})^{2} \mathrm{~h}^{2}+\left(\mathrm{V}_{\mathrm{T}} / \mathrm{h}\right)^{2} \mathrm{~h}^{2}\right\}+\eta_{2}(\mathrm{~V} / \theta \mathrm{h})^{2} \theta \mathrm{h}^{2}
$$

It may be remarked that other terms can occur, which are logarithms, but such terms can be considered not to change very much, therefore there are included in the constants. Anyway, we are only interested by order of magnitudes, and not accurate numbers, due to the approximations already made.

This dissipation is equilibrated by the driving power $\mathrm{P}$, due to interfacial tension, acting in favor of the minimization of the total area. Here, $P$ is given by [19]:

$$
\mathrm{P}=\mathrm{FV}=2 \sigma \mathrm{V} \cos \theta \approx 2 \sigma \mathrm{V}
$$

Letting $\varepsilon=\eta_{1} / \eta_{2}$ we then find:

$$
\frac{\sigma}{\eta_{1} \mathrm{~V}(\theta)} \approx \theta^{2}+\frac{1}{\varepsilon^{2} \theta^{2}}+\frac{1}{\varepsilon \theta}
$$

But the actual important parameter is $\dot{\mathrm{D}}$. After combining (12) and (17), $\dot{\mathrm{D}}$ is found to be related to $\theta$ and $\varepsilon$ by:

$$
\left|\frac{\sigma}{\eta_{1} \dot{\mathrm{D}}}\right| \approx \theta+\frac{1}{\varepsilon^{2} \theta^{3}}+\frac{1}{\varepsilon \theta^{2}}=\mathrm{G}(\theta)
$$

where $|\mathrm{A}|$ represents the absolute value of $\mathrm{A}$. 
Finding the maximum of $|\dot{D}|$ is then equivalent to finding the minimum of (18), in other words the minimum of $G(\theta)$. The function $G(\theta)$ is first decreasing, then it goes through a minimum at $\theta_{0}$ until it increases again with an asymptotic behavior $\mathrm{G}(\theta) \approx \theta$ (figure 8 ). The minimum is obtained for $\theta_{0}$ which is the solution of an equation of the fourth order and cannot be simplified. Let us look at the two limiting cases:

Assuming that $\varepsilon>1 / \theta_{0}$, i.e. $\eta_{1} / \eta_{2}>1 / \theta_{0}$ (neglecting the second term on the right side of (18)), we find that the minimum of (18) is obtained for $\theta_{0} \approx \varepsilon^{-1 / 3}$. In this case :

$$
\left|\frac{\sigma}{\eta_{1} \dot{D}}\right| \approx \varepsilon^{-1 / 3} \quad \text { and } \quad \mathrm{V}\left(\theta_{0}\right) \approx \frac{\sigma}{\eta_{1}} \varepsilon^{2 / 3}
$$

From the previous section, we found that $t_{c} \approx \theta_{0} R_{1} / V$ so that $\sigma t_{c} / \eta_{1} R_{1}$ is equivalent to $\frac{\sigma}{\eta_{1} \mathrm{~V}\left(\theta_{0}\right)}$, so that finally

$$
\frac{\sigma \mathrm{t}_{\mathrm{c}}}{\eta_{1} \mathrm{R}_{1}} \approx \varepsilon^{-2 / 3} \approx\left(\frac{\eta_{1}}{\eta_{2}}\right)^{-2 / 3}
$$

This slope is not -0.5 but close to it, and considering experimental errors, it may be concluded that it is in reasonable agreement with the experimental data.

The other case corresponds to $\varepsilon<1 / \theta_{0}$, when neglecting the third term on the right of (18), i.e. $\frac{\eta_{1}}{\eta_{2}}<1 / \theta_{0}$ and it is found that the minimum of (18) is obtained for $\theta_{0} \approx \varepsilon^{-1 / 2}$. Then

$$
\left|\frac{\sigma}{\eta_{1} \dot{\mathrm{D}}}\right| \approx \varepsilon^{-1 / 2} \quad \text { and } \quad \mathrm{V}\left(\theta_{0}\right) \approx \frac{\sigma}{\eta_{1}} \varepsilon
$$

In other words

$$
\frac{\sigma \mathrm{t}_{\mathrm{c}}}{\eta_{1} \mathrm{R}_{1}} \approx \varepsilon^{-1} \approx\left(\frac{\eta_{1}}{\eta_{2}}\right)^{-1}
$$


This gives an explanation of the first two slopes (regimes) obtained at very small and moderate viscosity ratios. Since we omitted the constants, it is not possible to determine the exact value of the limiting parameter $\frac{\eta_{1}}{\eta_{2}}$ at the change of regimes, but we may conclude that the order of magnitude is correctly prescribed.

We finally consider the case when the viscosity ratio $\frac{\eta_{1}}{\eta_{2}}$ is very large. In this situation, it is difficult to assume a particular type of flow. Therefore we may recall the solution by Frenkel [10], giving the growth of $x(t)$ when the outer fluid viscosity is small (i.e.

$\frac{\eta_{1}}{\eta_{2}}$ is very large). In fact this means that we neglect $\eta_{2}$. In this case, $x(t)$ is given by:

$$
x^{2}=\frac{3 R_{1} \sigma}{2 \pi \eta_{1}} t
$$

In addition to (23), inspection of figure 6 leads to the relating for $D(t)$ :

$$
D(t)=2 R+2 \sqrt{R^{2}-x^{2}}
$$

To first order in $\theta$, we can again assume that $\mathrm{R} \approx \mathrm{R}_{1}$. Thus :

$$
\mathrm{D}(\mathrm{t})=2 \mathrm{R}_{1}\left(1+\sqrt{1-\frac{3 \sigma \mathrm{t}}{2 \mathrm{R}_{1} \pi \eta_{1}}}\right)
$$

$F(t)$ now equals $D(t) / 4 R_{1}$. It is a decreasing function of time with no inflexion point, but its maximum slope is at $t=0$ so that $F^{\prime}(0)=-1 / t_{c}\left(t_{c}\right.$ is the point of maximum slope in this case), therefore we find a formula for the collision time such that :

$$
\frac{\sigma \mathrm{t}_{\mathrm{c}}}{\eta \mathrm{R}_{1}}=\frac{8 \pi}{3} \approx 8.4
$$


This value has the order of magnitude found previously and is in reasonable agreement with the data (8.4 compared to 12).

A possible improvement of the model is as follows. One needs to consider the exact flow field in the vicinity of the droplets. This will give rise to an exact calculation of the viscous dissipations and the interfacial tension driving power terms. They may actually involve not only shear effects but the elongational ones as well, because of the complexity of the flow involved. This problem will not be discussed here. It may also be considered numerically. Nevertheless, it can be concluded that this simple model is good enough to be able to predict the three regimes qualitatively, i.e. it is in reasonable agreement with the experiments.

\section{DISCUSSION}

\section{Results}

The procedure presented in this work is complementary to previous studies which are mostly devoted to studies of the initial phase of coalescence, in other words the drainage problem [8-9]. In particular, Chesters and coworkers have also recently studied numerically the importance of the dispersed to continuous-phase viscosity ratio $\eta_{1} / \eta_{2}$ on the drainage problem between interacting drops [20]. They have found limiting cases corresponding to partially mobile or immobile interfaces. The aim of the present work was not to discuss the three stages of the coalescence process, but on the other hand to look at the collision mechanism. As already discussed before, the viscosity ratio is the major parameter. It is shown here that limiting regimes are found which are independent of the small viscosity: when $\eta_{1} / \eta_{2}$ is small, the time of collision is proportional to $\eta_{2}$, the large viscosity. Similarly, when $\eta_{1} / \eta_{2}$ is large, $t_{c}$ is proportional to $\eta_{1}$, the large viscosity. From the experiments, the 
relation is given approximately by $t_{c} \cong 12 \eta R_{1} / \sigma$ (where $\eta$ is the large viscosity). Interestingly, the constant is the same in both cases, although there is no symmetry here. The first case is the one where one pushes a very viscous fluid, whereas the second one is related to motion of a very viscous fluid pushing a much less viscous one. Locally, at the neck, the velocity field is dominated by the interface velocities and all the dissipation comes from a very small region close to the apparent cusp. Therefore, we may explain this result by assuming that the local flow is the same in both cases, and that it controls the local dissipation. This is also the reason why the model of de Gennes and Brochard [18-19] is used here, for it assumes that the dissipation occurs only in a very small region close to the singular point. Most likely, the flow in such situations is close to a plug flow. Another interesting study would be to determine the exact shape of the velocity profile.

The problem of the singular point is also of concern, because one may argue that figures 6 and 7 are drawn as if there was a cusp. One may also refer to other similar works where cusps are discussed, like when pulling a fluid with a cylinder, half immersed in this fluid [21-22]. Indeed, Newtonian and non-Newtonian fluids are considered to allow this type of situation, going from a cusp to a gradually smooth curvature. In particular, asymptotic analyzes can allow to treat such problems, and also allow the effect of interfacial tension. In our case, we consider that the shape of the free surface at the moving boundary point $\mathrm{A}$ is initially a cusp, but after a very small time, it certainly corresponds to a small (compared to $\mathrm{R}_{1}$ ) but finite radius of curvature. This can also be observed from figures 1a-1f. Probably another important problem would be the close experimental analysis of the so-called cusp, especially at very small times.

Speaking about the theoretical part leads us to pose the question of the intermediate slope which is predicted by a power law of $-2 / 3$ instead of -0.5 experimentally. Nevertheless, it is likely that changing fluid 1 into 2 should not give the same result, except in the limiting 
cases where one viscosity is dominant. Generally speaking, the slope of $-2 / 3$ may seem quite reasonable. The errors on data points can allow one to think that a slope of $-2 / 3$ agrees also well with experimental points. Errors may be due to the fact that only approximately equal spheres are considered here, and that the influence of the radii ratio $R_{1} / R_{2}$ can affect some of the measurements.

\section{Perspectives}

This work leads to the natural improvement of this technique into an interfacial tensiometer for polymers. Such apparatuses are well known to physicists and are of different types. The most common ones available nowadays are based on the filament break-up technique [23], or the retraction of an ellipsoid [24], whereas the spinning drop tensiometer [25-26] or the usual pendant-drop technique [27] are older techniques but still give valuable information. The technique proposed here can be used as a measuring device as well. It requires a microscope only, which is available in most research laboratories or private research centers. The mixing of the fluids is quite easy and the only needed parameters are the viscosities at the required temperature. The only quantity to be measured is the collision time. This is probably better achieved using a video-camera with a well-defined speed (frame/second). In the experiment, $40 \mathrm{~ms}(1 / 25 \mathrm{~s})$ was the fastest time interval between the frames and it was fast enough for such fluids. It should be also perfectly adapted for highly viscous fluids like molten polymers.

One the major advantages of the technique is that sample preparation is quite easy, and mixing of the fluids is easily achieved using small amounts. This is much better than in other techniques like the filament break-up for example, which requires to mold a long regular filament inside another polymer. The amount of time required for the experiments is of course viscosity dependent and may turn out to be long, but one may use higher amounts of fluid 1 , in order to obtain many droplets. In such a system, one can then pick two very close droplets. 
Thus we limit the time to drain fluid 2, since the initial conditions correspond to small gaps or films. Once the collision process has started, it is only required to capture the first instants, as described, and this method has the advantage to be time-saving. It is not needed to way until final relaxation.

Temperature control may be one problem, if one wants to study (under a microscope) polymers which are in their molten state at high temperatures. Up to now, the author is only aware of heating systems to be operated with a microscope up to $100^{\circ} \mathrm{C}$. On the other hand, it is possible to design heating systems (up to $250^{\circ} \mathrm{C}$ ) to be operated under other visualization systems. The use of a macro lens coupled with a high resolution camera (1/3" size) can constitute an interesting solution.

The final question is also to relate the collision time with the interfacial tension. The answer is given by formulae (4a-c). Depending on the viscosities of the fluids, one will have to use one formula, or the others. This may be an advantage, because the use of a low viscosity fluids is convenient. Indeed, they are easier to handle and the corresponding collision times are shorter. Still one has to be careful about the molecular weight dependence of interfacial tension in polymeric systems, which may affect the results [27].

Another interesting aspect will be the study of the influence of the viscoelastic properties of the polymers on droplet collision. This has been introduced by a few authors [16] and an interesting comparison may be drawn. Finally, the influence of copolymers (type, triblock or diblock, architecture, length, etc.) at the interface is also another possible application for such a tensiometer. 


\section{CONCLUSION}

In this work, a simple experiment on droplet collision is presented. The effect of the viscosity ratio on the collision time has been considered with a wide range of fluids, covering about six decades of viscosity ratio. Three different regimes have been found experimentally. If the viscosity of one fluid (droplet or matrix) is much larger, then it governs collision. In the other intermediate case (viscosities in the same range), then the collision time varies approximately like the geometric mean of the viscosities, as observed previously.

Due to the very rapid growth of the neck, we have proposed a model considering the local viscous dissipations at the neck. This model gives a qualitative description of the viscosity ratio influence on the collision time.

Finally, an extension of this method to the design of a new interfacial tensiometer has been proposed, and its application for melts is possible. The influence of viscoelasticity and/or the influence of compatibilizers at the interface may also be interesting problems to consider with this technique.

\section{ACKNOWLEDGEMENTS}

The author is greatful to P.G. de Gennes and F. Brochard-Wyart for their helpful suggestions concerning the theoretical part. 


\section{REFERENCES}

[1] Taylor GI. Proc. Royal Soc. 1879; 29:501-523.

[2] Grace HP. Chem. Eng. Commun. 1982; 14: 225-277.

[3] Barthès-Biesel D, Acrivos A. J. Fluid. Mech. 1973; 61: 1-21.

[4] Acrivos A, Lo TS. J. Fluid Mech. 1978; 86: 641-672.

[5] Grizzuti N, Bifulco O. Rheol. Acta 1997; 36: 406-415.

[6] Vinckier I, Moldenaers P, Terracciano AM, Grizzuti N. AIChE J. 1998; 44: 951-958.

[7] Janssen JMH. "Dynamics of liquid-liquid mixing". PhD thesis. Eindhoven University of Technology. Eindhoven. The Netherlands, 1993.

[8] Chesters AK. Trans I Chem E. 1991; 69A: 259-270.

[9] Fortelny I, Zivny A. Polymer 1995; 36: 4113-4118.

[10] Frenkel J. J. Phys. USSR 1945; 9: 385-391.

[11] Kuczynski GC. Transactions AIME 1949; 185: 169-178.

[12] Hopper RW. J. Fluid Mech. 1990; 213: 349-375.

[13] Hopper RW. J. Fluid Mech. 1992; 243: 171-181.

[14] Eggers J. Phys. Rev. Letters 1998; 80: 2634-263.

[15] Verdier C. C. R. Acad. Sci. Paris Série IV 2000; 1: 119-126.

[16] Mazur S, Plazek DJ. Prog. Organic Coat. 1994; 24: 225-236.

[17] Sundararaj U, Macosko CW. Macromolecules 1995; 28: 2647-2657.

[18] Brochard-Wyart F, de Gennes PG. Advances Colloid Interface Sci. 1992; 39: 1-11.

[19] de Gennes PG, Brochard-Wyart F. 2000, unpublished result .

[20] Bazhlekov IB, Chesters AK, van de Vosse FN. Int. J. Multiphase Flow 2000; 26: 445466.

[21] Joseph DD, Nelson J, Renardy M, Renardy Y. J. Fluid Mech. 1991 ; 223 : 383-409. 
[22] Joseph DD. J. Non Newtonian Fluid Mech. 1992 ; 44: 127-148.

[23] Cohen A, Carriere CJ. Rheol. Acta 1989 ; 28: 223-232.

[24] Luciani A, Champagne MF, Utracki LA. J. Polym. Sci. B: Polym. Phys. 1997 ; 35: 13931403.

[25] Elmendorp JJ, de Vos G. Polym. Eng. Sci. 1986; 26(6): 415-417.

[26] Joseph DD, Arney MS, Gillberg G, Hu H, Hultman D, Verdier C, Vinagre TM. J. Rheol. 1992 ; 36(4): 621-662.

[27] Wu S, Polymer interface and adhesion, Marcel Dekker, 1982 (chapter 3). 


\section{FIGURE CAPTIONS}

Fig. 1 Collision of droplets of PIB (430 Pa.s) in PDMS (60 Pa.s)

a) $\mathrm{t}=0 \mathrm{~s}$ b) $\mathrm{t}=10 \mathrm{~s}$ c) $\mathrm{t}=10.8 \mathrm{~s}$ d) $\mathrm{t}=12.4 \mathrm{~s}$ e) $\mathrm{t}=16.8 \mathrm{~s}$ f) $\mathrm{t}=21.6 \mathrm{~s}$

Fig. 2 Schematic picture of the droplets and parameters

Fig. 3 Typical dimensionless length $D(t) / D_{0}$ vs. time $t$

Fig. 4 Experimental curve with equation fit to determine $m_{1}$ and $m_{2}$ with PIB (130 Pa.s) in PDMS (100 Pa.s). Best comparison obtained for $\mathrm{m}_{1}=7.24 \mathrm{~s}^{2}$ and $\mathrm{m}_{2}=0.61$

Fig. 5 Dimensionless collision time versus $\eta_{1} / \eta_{2}\left(T=25^{\circ} \mathrm{C}\right)$

Fig. 6 Geometry of the two-sphere model

Fig. 7 Sketch of the flow in the neck region

Fig. 8 Function $\mathrm{G}(\theta)$ 

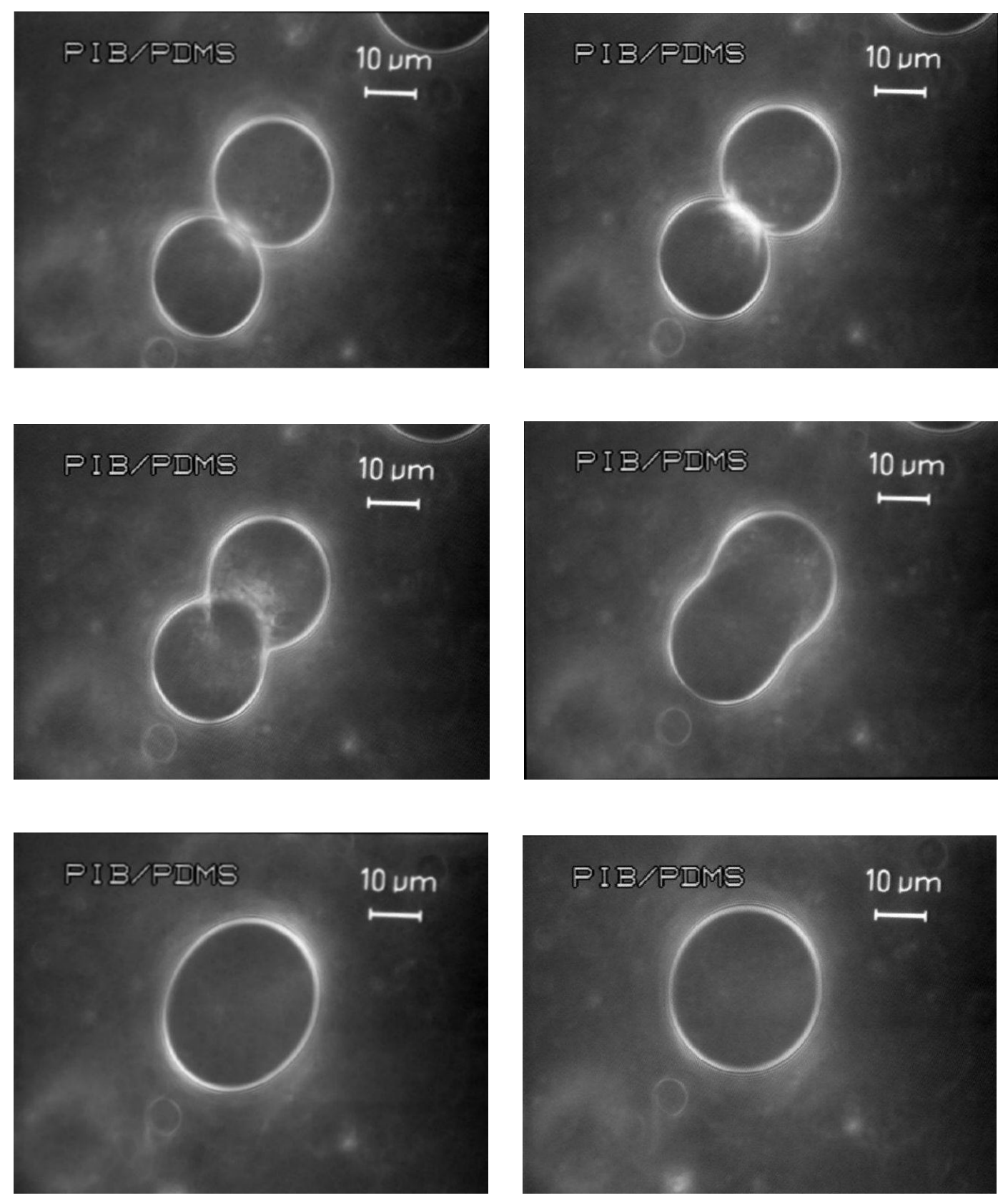

FIGURE 1 


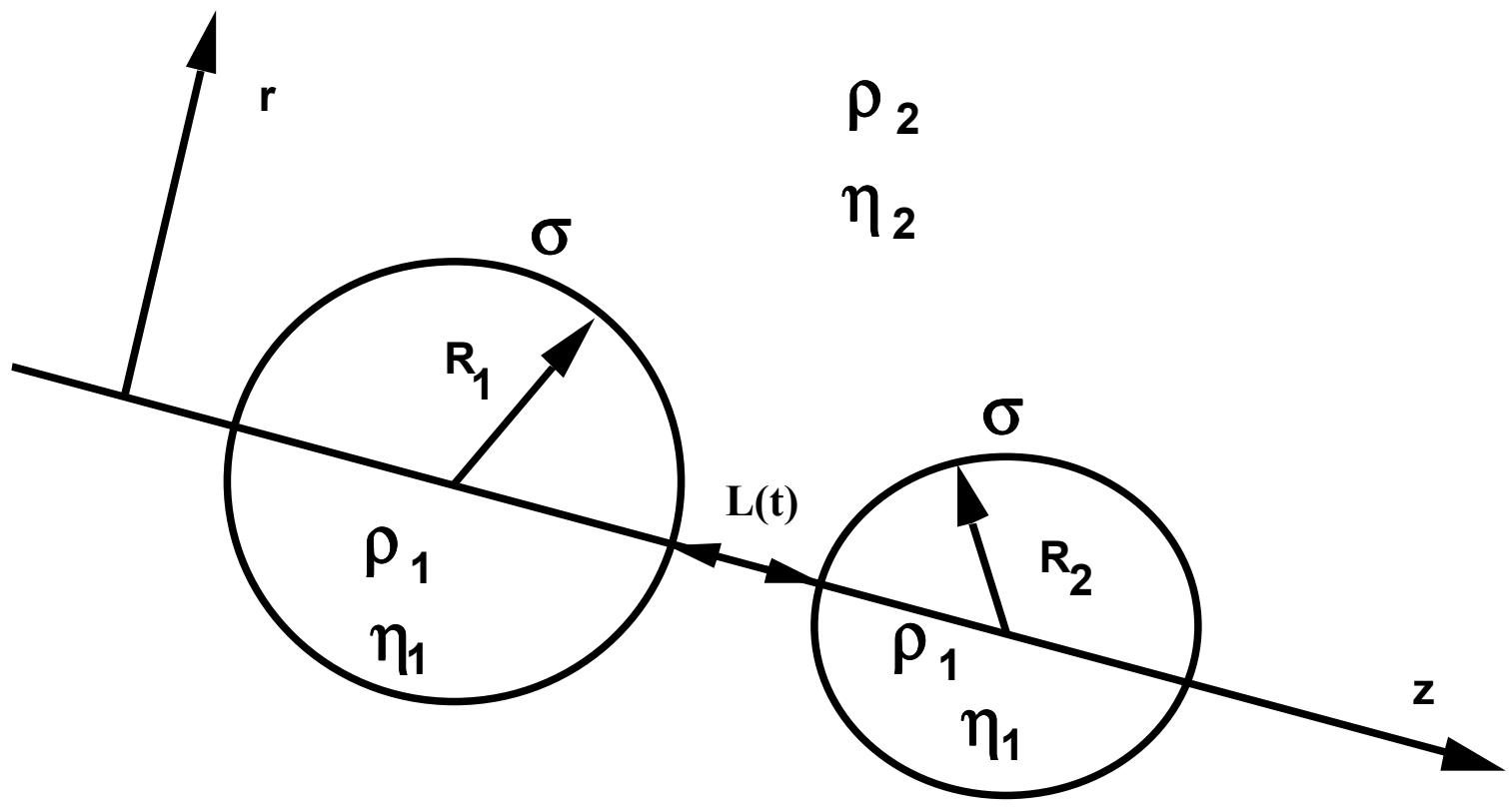

FIGURE 2 


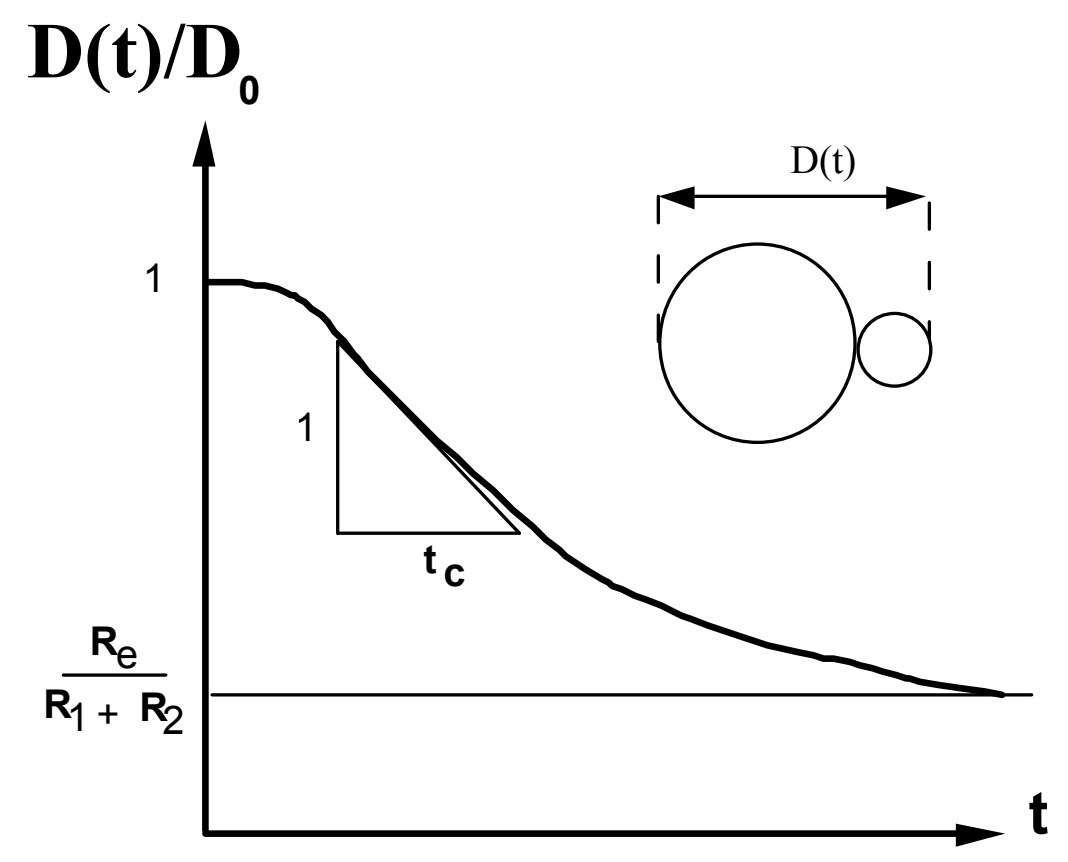

FIGURE 3 


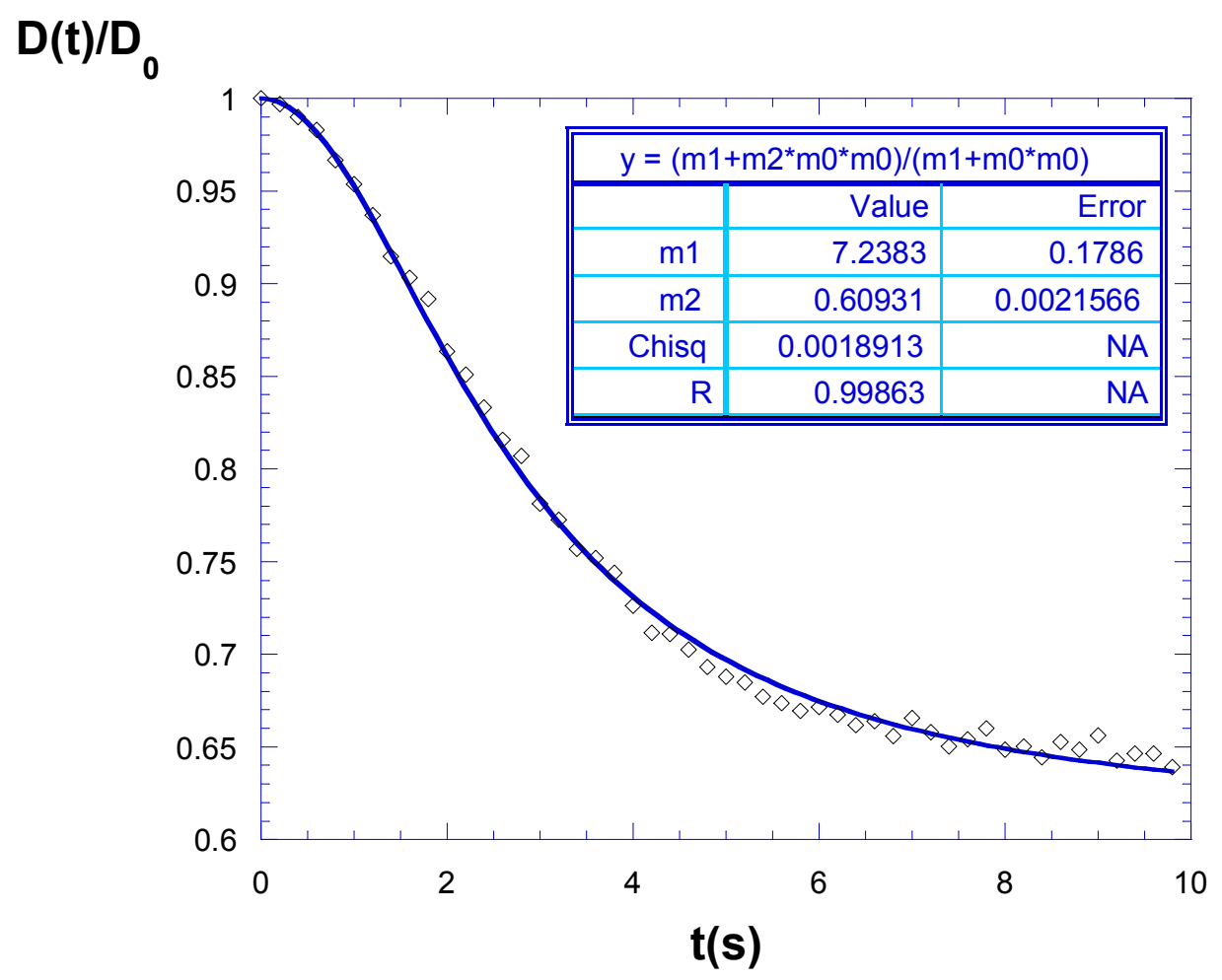

FIGURE 4 


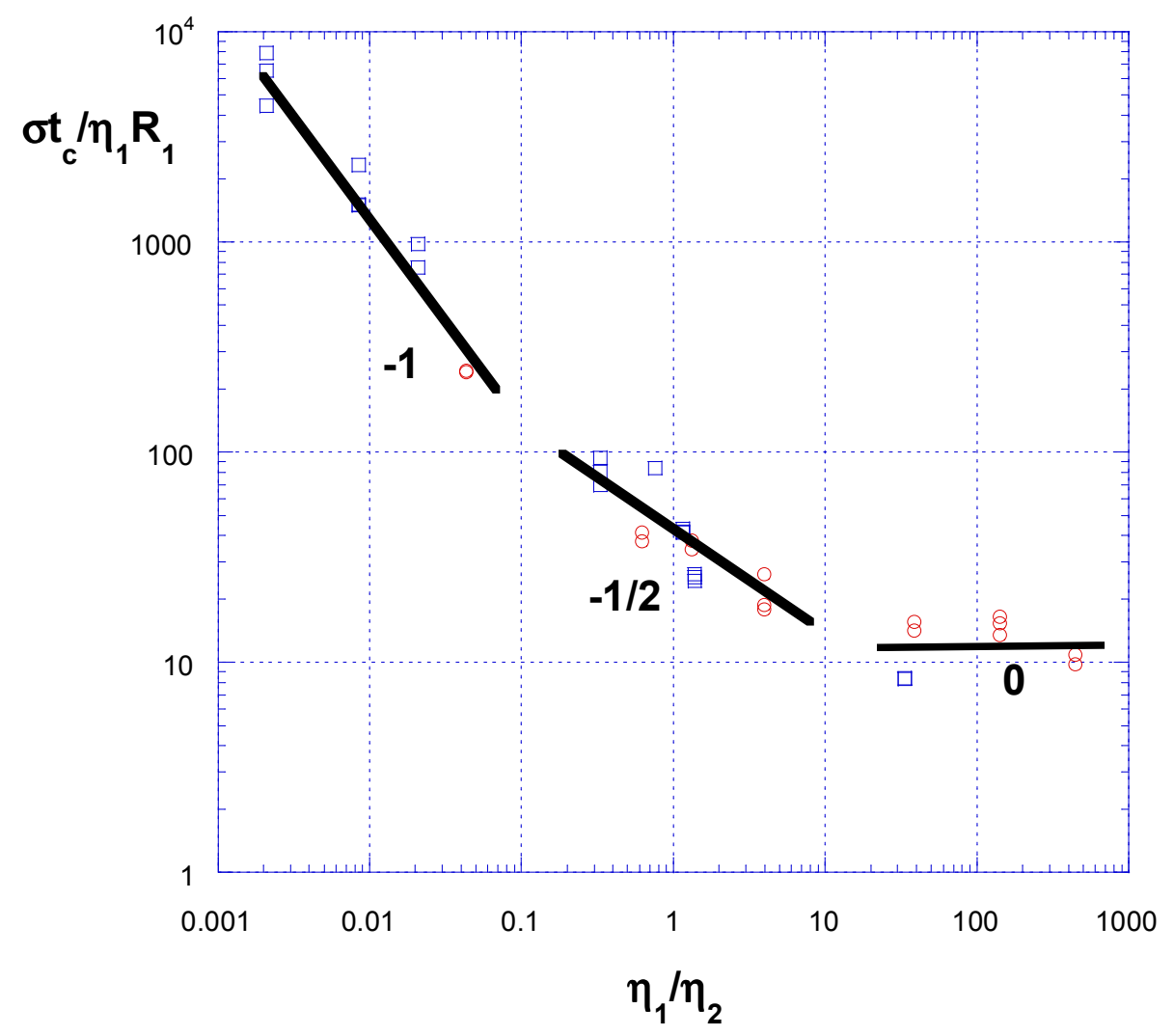

FIGURE 5 


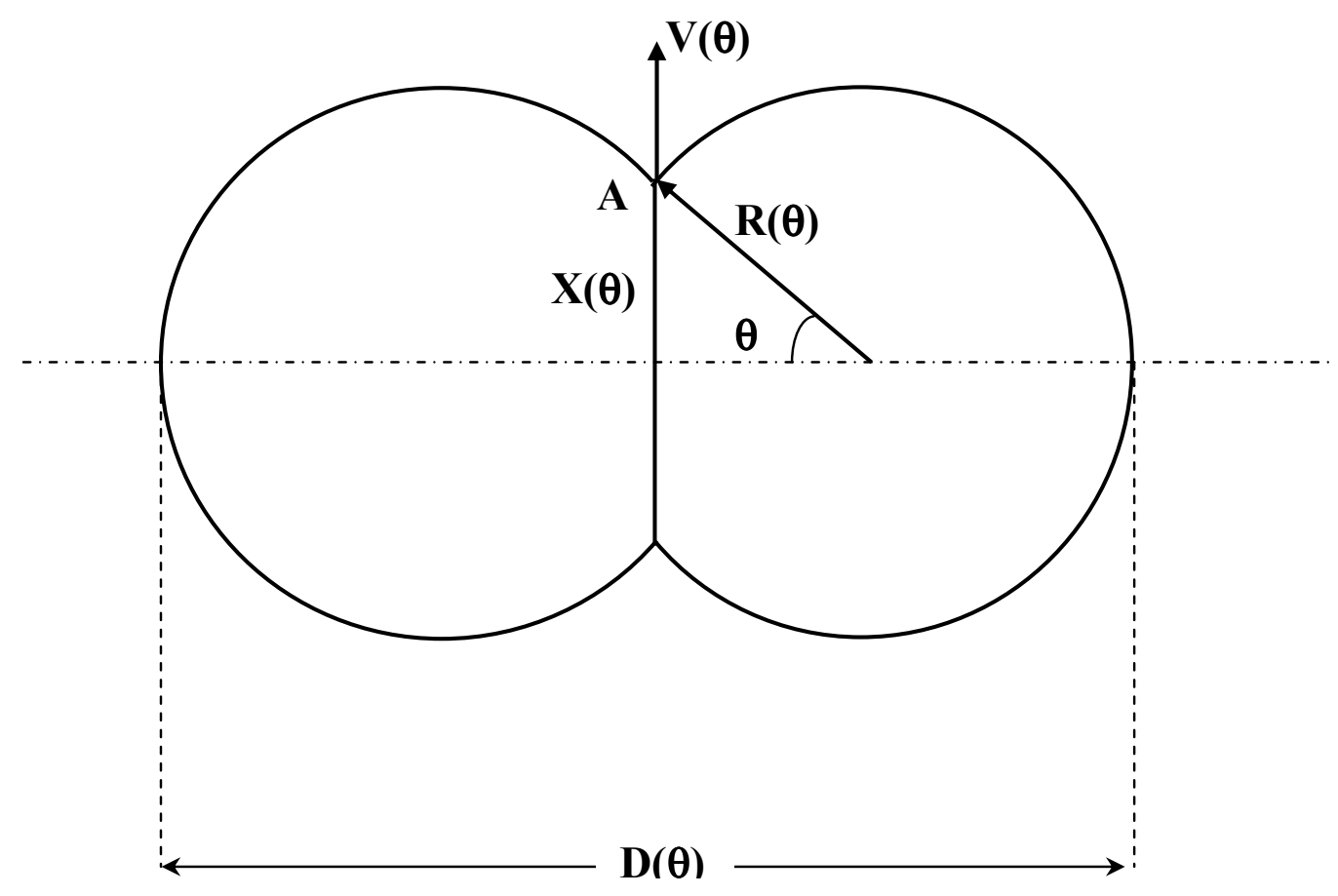

FIGURE 6 


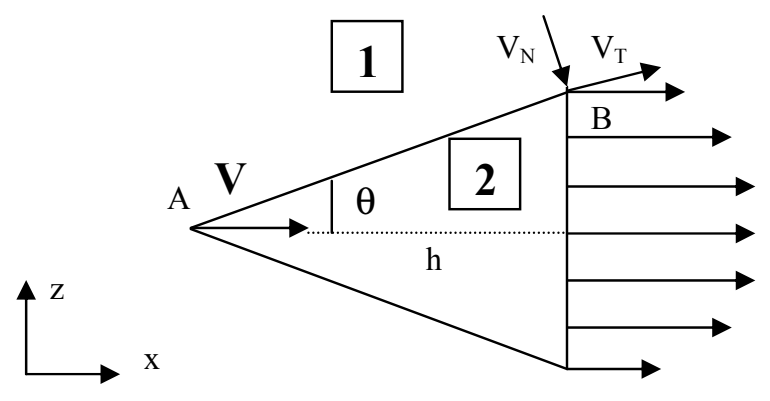

FIGURE 7 


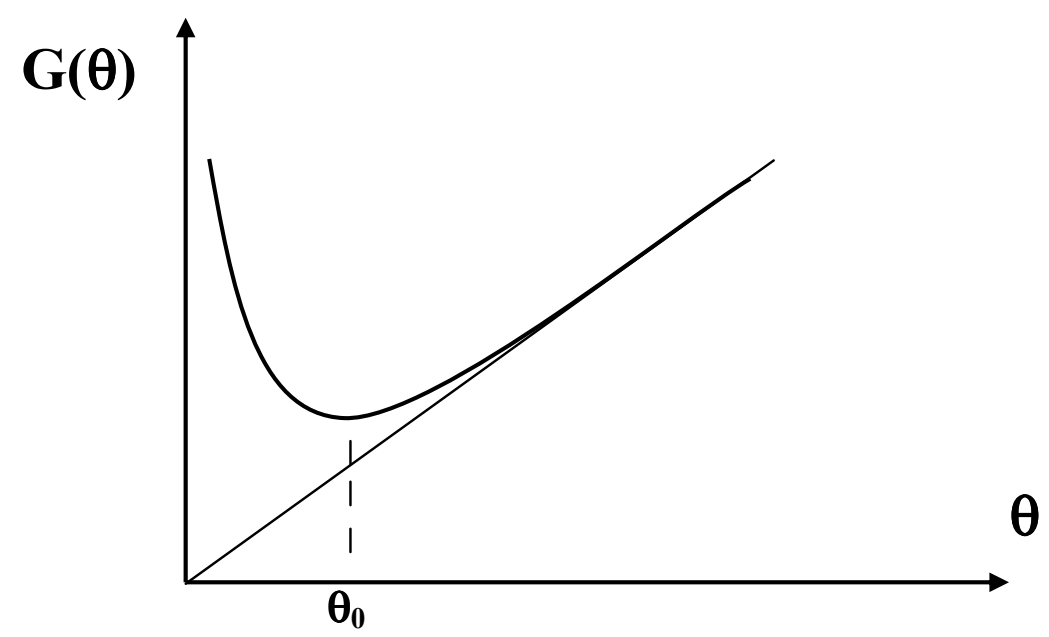

FIGURE 8 